\title{
Retinitis Pigmentosa with EYS Mutations Is the Most Prevalent Inherited Retinal Dystrophy in Japanese Populations
}

\author{
Yuuki Arai, ${ }^{1}$ Akiko Maeda, ${ }^{2,3}$ Yasuhiko Hirami, ${ }^{4}$ Chie Ishigami, ${ }^{1}$ Shinji Kosugi, \\ Michiko Mandai, ${ }^{1}$ Yasuo Kurimoto, ${ }^{6}$ and Masayo Takahashi ${ }^{1}$ \\ ${ }^{1}$ Laboratory for Retinal Regeneration, Center for Developmental Biology, RIKEN, Kobe 650-0047, Japan \\ ${ }^{2}$ Department of Ophthalmology, Case Western Reserve University, Cleveland, OH 44124, USA \\ ${ }^{3}$ Department of Pharmacology, Case Western Reserve University, Cleveland, OH 44124, USA \\ ${ }^{4}$ Institute of Biomedical Research Innovation Hospital, Kobe 650-0047, Japan \\ ${ }^{5}$ Department of Medical Ethics/Medical Genetics, Kyoto University School of Public Health, Kyoto 606-8501, Japan \\ ${ }^{6}$ Kobe City Medical Center General Hospital, Kobe 650-0047, Japan
}

Correspondence should be addressed to Akiko Maeda; aam19@case.edu

Received 7 January 2015; Accepted 19 May 2015

Academic Editor: Antonio Benito

Copyright (C) 2015 Yuuki Arai et al. This is an open access article distributed under the Creative Commons Attribution License, which permits unrestricted use, distribution, and reproduction in any medium, provided the original work is properly cited.

The aim of this study was to gain information about disease prevalence and to identify the responsible genes for inherited retinal dystrophies (IRD) in Japanese populations. Clinical and molecular evaluations were performed on 349 patients with IRD. For segregation analyses, 63 of their family members were employed. Bioinformatics data from 1,208 Japanese individuals were used as controls. Molecular diagnosis was obtained by direct sequencing in a stepwise fashion utilizing one or two panels of 15 and 27 genes for retinitis pigmentosa patients. If a specific clinical diagnosis was suspected, direct sequencing of disease-specific genes, that is, $A B C A 4$ for Stargardt disease, was conducted. Limited availability of intrafamily information and decreasing family size hampered identifying inherited patterns. Differential disease profiles with lower prevalence of Stargardt disease from European and North American populations were obtained. We found 205 sequence variants in 159 of 349 probands with an identification rate of $45.6 \%$. This study found 43 novel sequence variants. In silico analysis suggests that 20 of 25 novel missense variants are pathogenic. EYS mutations had the highest prevalence at 23.5\%. c.4957_4958insA and c.8868C > A were the two major EYS mutations identified in this cohort. EYS mutations are the most prevalent among Japanese patients with IRD.

\section{Introduction}

Retinitis pigmentosa (RP) is the most common form of inherited retinal dystrophies (IRD) and is clinically and genetically heterogeneous. At least 50 genes have been identified for nonsyndromic RP [1] (RetNet; http://sph.uth.tmc.edu/RetNet/ provided in the public domain by the University of Texas Houston Health Science Center, Houston, TX). They include genes required for phototransduction, visual cycle, cilial transportation in photoreceptors, and maintenance of photoreceptor structure $[2,3]$. RP patients commonly display a disease progression profile beginning with rod photoreceptor degeneration followed by cone photoreceptor death; hence patients with RP present clinically with night blindness and progressive restriction of the visual field followed by impairment of central and color vision. The prevalence of RP has been reported at 1 in 3,000-4,000 individulas worldwide [2] and a similar rate is expected in Japanese populations. Autosomal dominant (adRP), autosomal recessive (arRP), and X-linked (xlRP) patterns of inheritance are common in RP. Within Japanese populations identifiable inheritance patterns are recognized in nearly $50 \%$ of all RP cases with $35 \%, 10 \%$, and $5 \%$ in arRP, adRP, and xlRP, respectively. The remaining $50 \%$ of cases are considered simplex or sporadic. In the clinic, gaining information about a mode of inheritance is limited due to recent social trends including decreasing family size and increasing social isolation. Therefore, establishment of molecular diagnoses for patients with unknown disease inheritance is critical. We reported in 2008 the first comprehensive molecular diagnosis for RP 
in Japanese patients with known and unknown inheritance and identified 26 mutations in 28 of 209 probands (203 of RP, 2 of areolar atrophy, 3 of cone dystrophy, and 1 of Stargardt disease) [4]. To accomplish this study, we employed a method which combined an efficient denaturing high performance liquid chromatography (dHPLC) based assay with 108 exons of $30 \mathrm{RP}$-causing genes and confirmative direct DNA sequencing.

In the present study, we further increased the screening number of the genes and exons, and another cohort of 349 Japanese patients was examined to gain additional information about disease prevalence and to identify the responsible genes for IRD in Japanese populations. For this purpose, direct sequencing of stepwise analyses utilizing one or two panels of 15 and 27 genes was conducted for RP cases. Disease-specific genes were also analyzed on patients with other IRD.

\section{Materials and Methods}

2.1. Patients and Families. We performed mutation analysis in a cohort of Japanese patients with IRD, who visited the RP/Genetic Counseling Clinic in the Institute of Biomedical Research and Innovation Hospital, Kobe, Japan, from October 2008 to May 2014. A total of 412 individuals, 349 probands, and their 26 affected and 37 unaffected family members were involved in this study. The Human Genetic Variation Database from 1,208 Japanese individuals provided by Japanese genetic variation consortium, Kyoto University, Kyoto, Japan, was used as controls (http://www.genome.med.kyoto-u.ac.jp/SnpDB/) [5]. None of the patients in this cohort were enrolled in our previous study [4]. Informed consent was obtained from all patients and family members after the genetic screening procedures had been fully explained. Research protocols were approved by the institutional review boards of the RIKEN and the local research ethics committees, and the study was conducted in accordance with the principals of the Declaration of Helsinki.

2.2. Mutation Analysis. Genomic DNA was extracted from peripheral lymphocytes using standard procedures. Mutation screening was performed by a stepwise direct sequencing utilizing one or two panels of genes listed in Supplemental Tables S1(A) and S1(B) in Supplementary Material available online at http://dx.doi.org/10.1155/2015/819760 for patients with RP (Figure 1). Only patients whose sequence variants were not detectable underwent additional screening with the 2 nd panel. If a specific clinical diagnosis was suspected, direct sequencing of disease-specific genes was conducted by analyzing the following genes for mutations (Supplemental Table S1(C)): $A B C A 4$ and $R D S / P R P H 2$ for Stargardt disease, CHM for choroideremia, CYP4V2 for Bietti crystalline dystrophy, $S A G$ for Oguchi disease, VMD2 for Best disease, USH2A for Usher syndrome, RS1 for retinoschisis, RDS/ $P R P H 2$ for central areolar choroidal dystrophy, and $R D H 5$ and $R L B P 1$ for fundus albipunctatus. In silico analysis was performed to evaluate the potential deleterious effects of novel missense mutations utilizing the following four

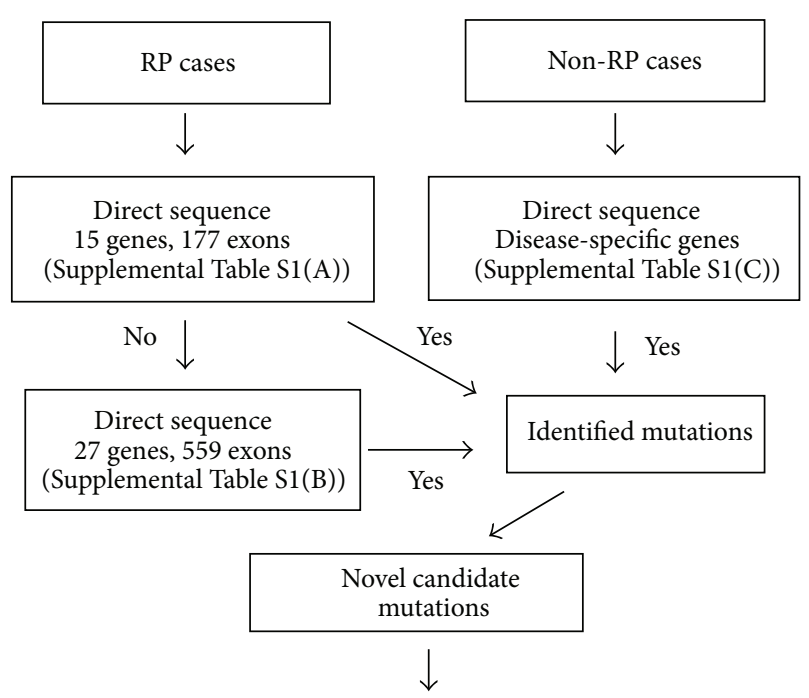

Validation, in silico analysis, and segregation analysis

FIGURE 1: A stepwise screening for patients with IRD. Molecular diagnosis was performed with a stepwise screening methodology. Patients with RP were initially screened with 15 genes, and additional 27 genes were sequenced when the initial screening failed to detect mutations. Disease-specific genes were sequenced for patients with other IRD.

computational prediction algorithms: PolyPhen2 (http:// genetics.bwh.harvard.edu/pph2/index.shtml), SIFT (http:// sift.jcvi.org/), PMut (http://mmb2.pcb.ub.es:8080/PMut/ PMut.jsp), and SNAP (https://rostlab.org/services/snap/). Variants were determined to carry potential deleterious effects when $50 \%$ and higher rates of the programs predict their pathogenicity. Segregation analysis was also applied to families with newly identified mutations.

2.3. Clinical Diagnosis. Full medical and family histories were taken, pedigrees were drawn, and ophthalmologic examinations were performed for each patient. Clinical evaluation included best correct visual acuity (BCVA) according to projected Snellen charts, slit-lamp biomicroscopy, and dilated indirect ophthalmoscopy. Retinal imaging using a Topcon TRC-NW7SF retinal camera (Topcon Corporation, Tokyo, Japan), optical coherence tomography, and retinal autofluorescence imaging using a SPECTRALIS_Spectral domain optical coherence tomography (OCT) scanner (Heidelberg Engineering, Heidelberg, Germany) were conducted. Fullfield electroretinogram (ERG) was also performed. The ERG protocol complied with the standards published by the International Society for Clinical Electrophysiology of Vision. Visual fields were examined with Goldmann perimetry.

\section{Results and Discussion}

3.1. A High Rate of Clinical Diagnosis as RP in This IRD Cohort. The summary of clinical diagnoses in our cohort is shown in Figure 2, and 313 of 349 (89.6\%) patients were diagnosed as nonsyndromic RP. The fraction of Stargardt disease patients 


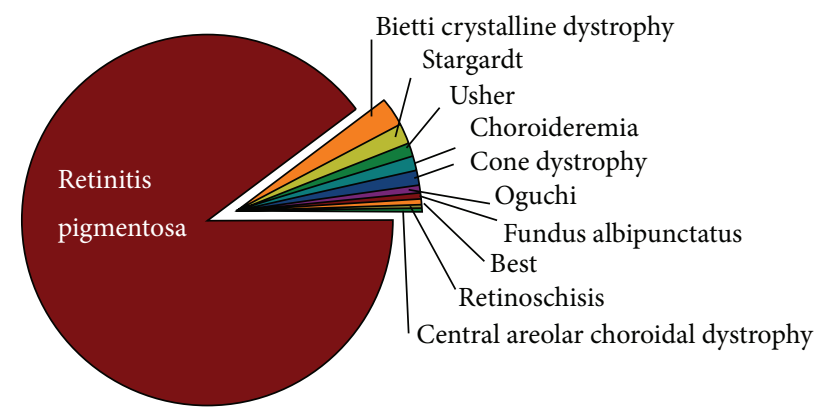

FIgURE 2: Retinal dystrophies included in this study. Clinical diagnosis of each retinal disease is shown. Nonsyndromic RP was found in 313 of 349 cases at the rate of $89.6 \%$. We revised the clinical diagnosis in one case after genotyping: the patient diagnosed with cone dystrophy was revised to autosomal recessive enhanced S-cone syndrome (ESC) due to compound heterozygous NR2E3 mutations: c. $419 \mathrm{~A}>\mathrm{G}$ and c. $488 \mathrm{~T}>\mathrm{C}$.

in our cohort was $1.2 \%$. The estimated prevalence of Stargardt disease is 1 in 8,000-10,000 individulas in North America [6] and that of RP is 1 in 3,000-4,000 individulas [2]. The estimated prevalence of RP in Japan is also 1 of 3,000-4,000 individulas; however, our data indicates a higher rate of RP and a lower prevalence of Stargardt disease in Japanese IRD populations. Additionally choroideremia, in which we identified 5 genetically unrelated patients in this study, has a reported prevalence of 1 in 50,000-100,000 individulas in North America (http://ghr.nlm.nih.gov/condition/choroideremia). Usher syndrome was found in 5 unrelated patients and was the only syndromic disease identified. Bietti crystalline dystrophy (BCD) is known to be more common in people with East Asian ancestry [7], and 11 unrelated BCD patients were identified in this cohort. Although the high RP and low Stargardt disease prevalence might be associated with patient referral bias to our clinic, the RP/Genetic Counseling Clinic, the current study clearly demonstrates differential disease profiles between racial backgrounds. We revised the clinical diagnosis in one case after genotyping: the patient diagnosed with cone dystrophy was revised to autosomal recessive enhanced S-cone syndrome (ESC) due to compound heterozygous NR2E3 mutations: c.419A $>\mathrm{G}$ and c.488T $>$ C.

\subsection{High Prevalence of EYS Mutations in Japanese Cases.}

Genetic testing is recommended for patients with IRD, because the results gained can make a positive impact on both patients and their families [8]. Identification of the causative genes leads to improved accuracy of diagnoses, providing patients prognostic information and better genetic counseling, and can facilitate further research in the development of mechanism-specific care. In this study we analyzed genetic mutations using the stepwise direct sequencing of the majority of coding sequence in genes which are known to cause RP and other IRD (Figure 1 and Supplemental Tables S1(A) and S1(B)). Only patients whose sequence variants were not detectable underwent additional screening with the 2 nd panel. If a specific clinical diagnosis was suspected, direct sequencing of disease-specific genes was conducted by
TABLE 1: Inherited patterns and diagnostic rates.

\begin{tabular}{lccc}
\hline Inheritance & Tested $(n)$ & Detected $(n)$ & Detected $(\%)$ \\
\hline Autosomal dominant & 35 & 21 & 60.0 \\
Autosomal recessive/simplex & 303 & 134 & 44.2 \\
X-linked & 11 & 4 & 36.3 \\
\hline & 349 & 159 & 45.6 \\
\hline
\end{tabular}

analyzing the following genes for mutations (Supplemental Table S1(C)).

Inherited patterns of IRD in this cohort are summarized in Table 1. A total of 205 changes in 26 genes were detected as summarized in Table 2 . These 205 sequence variants were found in 159 of 349 probands (45.3\%). These patients carried between 1 and 4 sequence variants. Surprisingly, 134 of 303 or $44.2 \%$ of autosomal recessive or simplex cases were identified to carry sequence variants, and this rate was higher than that of X-linked retinal dystrophies with the detection rate of $36.3 \%$. The identification rate of xlRP was reported as 35\% in cohorts of North American populations [9]. This study was designed and conducted as a continuation of our previous study which utilized a dHPLC based assay [4], and we also expanded the members of genes and exons examined. These changes successfully contributed to a better identification rate as compared to our previous study from $13.4 \%$ to $45.3 \%$.

Sequence variants in $E Y S$ were the most frequent in our cohort and were detected in 82 of 349 probands (23.5\%). The second most frequently mutated gene in our cohort was RDS/PRPH2 and accounted for $4.6 \%$ of cases. Mutations in EYS were found in 32.8\% [10] and 16\% [11] of Japanese arRP patients. Patients with compound heterozygous EYS mutations were summarized in Supplemental Table S2. EYS was identified in Spanish patients with arRP in 2008 [12], and indeed EYS was not tested in our 2008 study. The detection rate of this current study would drop to $20.6 \%$ if $E Y S$ was excluded from our current analysis. As compared to recently published Japanese cohort studies [13,14], this study displayed a lower detection rate of USH2A. This is probably due to our two-panel screening methodology. Since USH2A is included in the 2nd panel, this gene was not examined if the 1st panel detected sequence variation(s). Therefore, data interpretation needed to be performed cautiously and special consideration had to be made to the strengths and limitations of each method.

Another significant finding is that this study found a mutation c.469G > A (p.G157R) in GUCA1B in 4 patients with RP. The mutation in GUCA1B was found only in 3 Japanese adRP families [15] and no mutations in GUCA1B have ever been found in 400 British patients with adRP [16]. A more recent study failed to detect GUCA1B mutations in patients with cone dystrophy and cone-rod dystrophy, and thus the authors concluded that GUCA1B is a minor cause for IRD in Europeans and North Americans [17]. Given the result of 4 unrelated RP patients carrying the previously reported c. $469 \mathrm{G}>\mathrm{A}$ mutation in this cohort, this mutation could be frequent at least for the Japanese IRD populations. 
TABLE 2: Prevalence of mutations among 349 probands in this study.

\begin{tabular}{|c|c|c|c|c|}
\hline Gene & Location & Probands $(n)$ & Prevalence $(\%)$ & Disease category \\
\hline EYS & $6 \mathrm{q} 12$ & 82 & 23.5 & $\operatorname{arRP}$ \\
\hline RDS/PRPH2 & $6 \mathrm{p} 21.1$ & 16 & 4.6 & adRP, arRP, adMD ${ }^{*}$ \\
\hline RHO & $3 \mathrm{q} 22.1$ & 7 & 2.0 & adRP, arRP \\
\hline CYP4V2 & $4 \mathrm{q} 35.2$ & 7 & 2.0 & Bietti crystalline dystrophy \\
\hline$C R B 1$ & $1 \mathrm{q} 31.3$ & 5 & 1.4 & arRP, LCA \\
\hline$R P 11$ & $19 q 13.42$ & 4 & 1.2 & $\operatorname{adRP}$ \\
\hline GUCA1B & $6 \mathrm{p} 21.1$ & 4 & 1.2 & $\operatorname{adRP}$ \\
\hline PROM1 & $11 q 12.3$ & 4 & 1.2 & $\operatorname{adRP}$ \\
\hline$R P G R$ & Xp11.4 & 4 & 1.2 & $\mathrm{xlRP}$ \\
\hline$A B C A 4$ & $1 \mathrm{p} 22.1$ & 3 & 0.9 & $\mathrm{STGD}^{\dagger}$ \\
\hline ROM1 & $11 q 12.3$ & 3 & 0.9 & adRP, arRP \\
\hline$C R X$ & $19 \mathrm{q} 13.32$ & 2 & 0.6 & adRP, CORD ${ }^{\ddagger}$ \\
\hline $\mathrm{CHM}$ & $\mathrm{Xq} 21.2$ & 2 & 0.6 & Choroideremia \\
\hline$G U C Y 2 D$ & $17 \mathrm{p} 13.1$ & 2 & 0.6 & $\operatorname{arRP}$ \\
\hline$R P 2$ & Xp11.23 & 2 & 0.6 & $\mathrm{xlRP}$ \\
\hline$R P 9$ & $7 \mathrm{p} 14.3$ & 2 & 0.6 & adRP \\
\hline TOPORS & $9 \mathrm{p} 21.1$ & 2 & 0.6 & $\operatorname{adRP}$ \\
\hline USH2A & $1 \mathrm{q} 41$ & 2 & 0.6 & $\operatorname{arRP}$ \\
\hline CNGB3 & $8 \mathrm{q} 21.3$ & 1 & 0.3 & Cone dystrophy \\
\hline$I M P D H 1 / R P 10$ & $7 q 32.1$ & 1 & 0.3 & adRP \\
\hline$M A K$ & $6 \mathrm{p} 24.2$ & 1 & 0.3 & $\operatorname{arRP}$ \\
\hline NR2E3 & $15 \mathrm{q} 23$ & 1 & 0.3 & $\mathrm{ESC}^{\S}$ \\
\hline RDH5 & $12 \mathrm{q} 13.2$ & 1 & 0.3 & $\mathrm{FA}^{\#}$ \\
\hline$R P 1$ & $8 \mathrm{q} 12.1$ & 1 & 0.3 & adRP, arRP \\
\hline$R L B P 1$ & $15 q 26.1$ & 1 & 0.3 & arRP \\
\hline$S A G$ & $2 \mathrm{q} 37.1$ & 1 & 0.3 & Oguchi disease \\
\hline
\end{tabular}

205 gene alterations were found in 349 probands.

${ }^{*}$ MD: macular dystrophy.

${ }^{\dagger}$ STGD: Stargardt disease.

${ }^{\ddagger}$ CORD: cone-rod dystrophy.

${ }^{\S}$ ESC: enhanced S-cone syndrome.

${ }^{\#}$ FA: fundus albipunctatus.

3.3. Novel Sequence Variants Identified in this Study. This study revealed 43 novel sequence variants including 32 EYS mutations (Tables 3-5). A total of 11 novel sequence variants were identified in the following genes: $A B C A 4$, CRX, PROM1, RDS/PRPH2, RHO, and RP11 (Table 3). The same novel alteration, c.613_615delTAC in $R P 11$, was found in two unrelated families. As shown in Figure 3, segregation analyses revealed that this novel mutation contributed to adRP in this family with III-2 as a nonpenetrant. The same novel c.1738A $>C$ alteration was detected in PROM1 in three unrelated families (this variant is described more in the section of "Association of EYS, CRB1, and PROM1 in retinal dystrophy"). This cohort also included a family with a novel RHO sequence variant, c.36delC (Figure 4). II-5 in this family carried the heterozygous mutation, and her clinical phenotype was relatively mild with late onset at the age of 62 . III-2 showed only marginal clinical signs of RP when she had underwent clinical evaluations at the age of 44 . A possible carrier of this mutation, I-1, died before the age when III-2 presented RP symptoms. This truncating variant is located in exon 1 and it is likely to contribute to adRP. A total of 13 novel truncating EYS sequence variants were found in $21 \mathrm{RP}$ patients in 13 families (Table 4) and 19 missense changes in EYS were also recognized (Table 5). Two novel EYS mutations, c.8439_8442dupTGCA and c.5202_5203delGT, were found in a single family (Figure 5). Affected family members II-2 and II-4 carried the compound heterozygous mutations. In silico analysis suggests that 20 of 25 novel missense mutations identified here harbor potential deleterious effects (Table 5), and further segregation analyses are essential to conclude their pathogenesis.

3.4. c.4957_4958insA and c.8868C>A Are the Two Major EYS Mutations in Japanese Patients with RP. EYS alterations were detected in 126 alleles in 82 probands with the highest prevalence in this Japanese cohort of IRD (Table 2). All EYS sequence variants were found in patients with nonsyndromic RP. We found 4 major EYS variants which were detected in unrelated families. Mutations of c.4957_4958insA in exon 26 and c.8868C $>\mathrm{A}$ in exon 44 were the two highest 


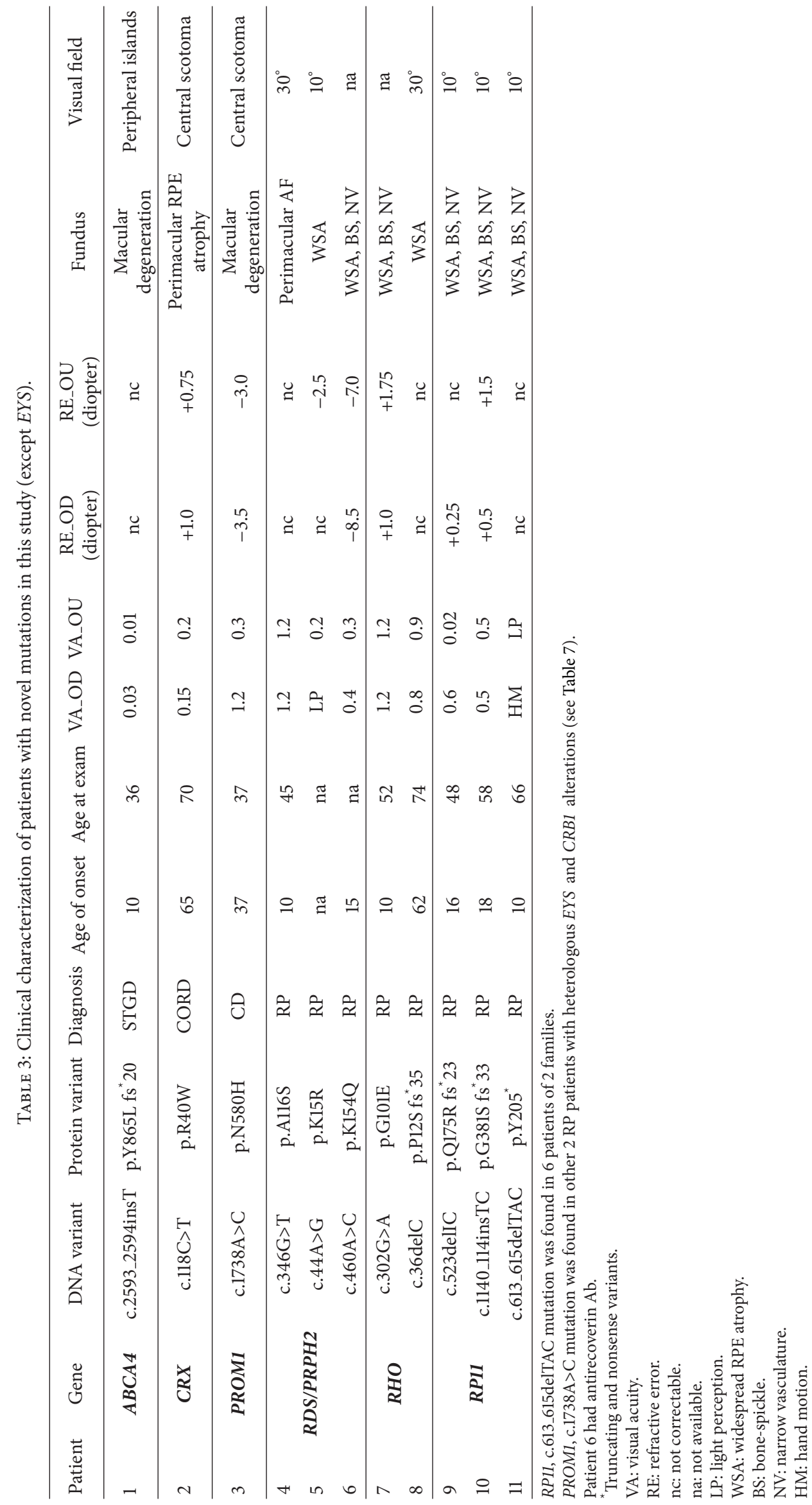


TABLE 4: Novel truncating and nonsense EYS mutations found in this study.

\begin{tabular}{|c|c|c|c|}
\hline Mutation & DNA variant & Protein variant & Diagnosis \\
\hline 1 & c.179delT & p.L60W fs 3 & $\mathrm{RP}$ \\
\hline 2 & IVS27-3_4insT & - & $\mathrm{RP}$ \\
\hline 3 & IVS38-1G>T & - & $\mathrm{RP}$ \\
\hline 4 & c. $2380 \mathrm{C}>\mathrm{T}$ & p.R794 & $\mathrm{RP}$ \\
\hline 5 & c. 4557 delA & p.A1520P fs 30 & $\mathrm{RP}$ \\
\hline 6 & c.5202_5203delGT & p.F1735Q fs 6 & $\mathrm{RP}$ \\
\hline 7 & c.6869_6896delCCATATTCCTGCAAATGTTCAAATTGATAAGAAAG & p.P2290Q fs 12 & $\mathrm{RP}$ \\
\hline 8 & c.6897_6902dupAGGTCC & p.G2300_P2301dup & $\mathrm{RP}$ \\
\hline 9 & c. $6976 \mathrm{C}>\mathrm{T}$ & p.R $2326^{*}$ & $\mathrm{RP}$ \\
\hline 10 & c.7836_7837delTC & p.P2613L fs ${ }^{*} 18$ & $\mathrm{RP}$ \\
\hline 11 & c.8196_8200delCTTTC & p.F2733C fs 33 & $\mathrm{RP}$ \\
\hline 12 & c.8439_8442dupTGCA & p.E2815C fs ${ }^{*} 19$ & $\mathrm{RP}$ \\
\hline 13 & c. $8921 \mathrm{C}>\mathrm{A}$ & p.S2974* & $\mathrm{RP}$ \\
\hline
\end{tabular}

TABLE 5: In silico analysis for novel missense mutations.

\begin{tabular}{|c|c|c|c|}
\hline Gene & Protein variant & DNA variant & $\begin{array}{l}\text { Prediction } \\
\text { for damage }\end{array}$ \\
\hline$C R X$ & p.R40W & c. $118 \mathrm{C}>\mathrm{T}$ & 4 of 4 \\
\hline EYS & p.K4E & c. $10 \mathrm{~A}>\mathrm{G}$ & 2 of 4 \\
\hline$E Y S$ & p.R26Q & c. $77 \mathrm{G}>\mathrm{A}$ & 2 of 4 \\
\hline EYS & p.M12T & c. $35 \mathrm{~T}>\mathrm{C}$ & 2 of 4 \\
\hline$E Y S$ & p.E47D & c. $141 \mathrm{~A}>\mathrm{T}$ & 3 of 4 \\
\hline$E Y S$ & p.Q76H & c. $228 \mathrm{G}>\mathrm{C}$ & 2 of 4 \\
\hline$E Y S$ & p.C211Y & c. $632 \mathrm{G}>\mathrm{A}$ & 4 of 4 \\
\hline EYS & p.I256M & c. $768 \mathrm{~A}>\mathrm{G}$ & 2 of 4 \\
\hline$E Y S$ & p.G484R & c. $1450 \mathrm{G}>\mathrm{A}$ & 3 of 4 \\
\hline$E Y S$ & p.N1205T & c. $3614 \mathrm{~A}>\mathrm{C}$ & 2 of 4 \\
\hline$E Y S$ & p.K1633E & c. $4897 \mathrm{~A}>\mathrm{G}$ & 2 of 4 \\
\hline EYS & p.L1655M & c. $4963 \mathrm{~T}>\mathrm{A}$ & 2 of 4 \\
\hline EYS & p.L1802F & c. $5404 \mathrm{C}>\mathrm{T}$ & 2 of 4 \\
\hline EYS & p.G2186E & c. $6557 \mathrm{G}>\mathrm{A}$ & 2 of 4 \\
\hline EYS & p.I2188T & c. $6563 \mathrm{~T}>\mathrm{C}$ & 1 of 4 \\
\hline$E Y S$ & p.R2604C & c. $7810 \mathrm{C}>\mathrm{T}$ & 2 of 4 \\
\hline$E Y S$ & p.T2683I & c. $8048 \mathrm{C}>\mathrm{T}$ & 0 of 4 \\
\hline$E Y S$ & p.D2767H & c. $8299 \mathrm{G}>\mathrm{C}$ & 3 of 4 \\
\hline$E Y S$ & p.L2784R & c. $8351 \mathrm{~T}>\mathrm{G}$ & 2 of 4 \\
\hline$E Y S$ & p.I3091T & c. $9272 \mathrm{~T}>\mathrm{C}$ & 2 of 4 \\
\hline PROM1 & p.N580H & c. $1738 \mathrm{~A}>\mathrm{C}$ & 3 of 4 \\
\hline$R D S$ & p.K15R & c. $44 \mathrm{~A}>\mathrm{G}$ & 1 of 3 \\
\hline$R D S$ & p.A116S & c. $346 \mathrm{G}>\mathrm{T}$ & 0 of 4 \\
\hline$R D S$ & p.K154Q & c. $460 \mathrm{~A}>\mathrm{C}$ & 0 of 4 \\
\hline $\mathrm{RHO}$ & p.G101E & c. $302 \mathrm{G}>\mathrm{A}$ & 4 of 4 \\
\hline
\end{tabular}

Bold indicates in silico analysis indicates pathogenic higher than $50 \%$ rates.

prevalent EYS mutations observed (Table 6). The frequencies of c.4957_4958insA and c.8868C>A variants were $26.8 \%$ (44 alleles in 82 probands with EYS mutations) and $13.4 \%$
(I)

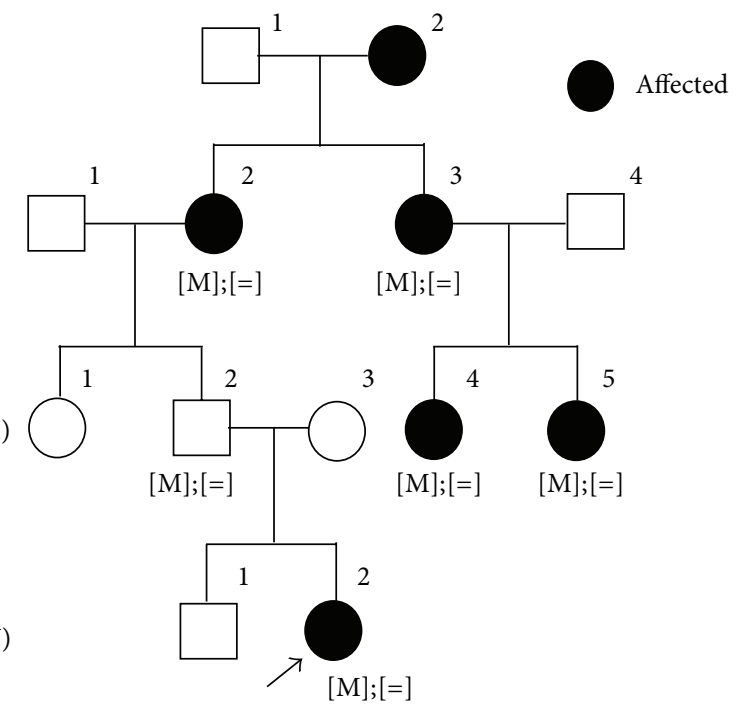

RP11 ex7 c.613_615delTAC

FIGURE 3: A pedigree of adRP with the novel RP11 mutation. A family carrying a novel c.613_615delTAC mutation in RP11 is presented. All of 6 family members who underwent molecular diagnosis carried the heterozygous c.613_615delTAC mutation as indicated with $[\mathrm{M}] ;[=]$. Affected individuals are indicated as filled symbols, and an arrow indicates the proband in this family.

(22 of 82), respectively. No c.4957_4958insA mutation has been detected in 1,208 Japanese individuals without eye symptoms. These data suggest that screening and developing treatment options for these two mutations, c.4957_4958insA and c.8868C $>$ A, can greatly improve RP care in Japan.

EYS was previously known as the RP25 gene located at a $16 \mathrm{cM}$ region on chromosome 6p12.1-q15 [18], and linkage to the same locus was reported in multiple families from various ancestral origins including Spanish [18], Pakistani [19], and Chinese [20]. The RP25 was identified as EYS in Spanish patients with arRP in 2008 [12]. Additional studies 
TABLE 6: Frequency of major EYS variants in 82 probands with EYS mutations.

\begin{tabular}{|c|c|c|c|c|c|}
\hline & Exon & DNA variant & Protein variant & Allele & Frequency \\
\hline EYS & ex26 & c.4957_4958insA & p.S1653K fs 2 & 44 & 26.8 \\
\hline$E Y S$ & ex32 & c. $6557 \mathrm{G}>\mathrm{A}$ & p.G2186E & 7 & 4.3 \\
\hline EYS & ex32 & c. $6563 \mathrm{~T}>\mathrm{C}$ & p.I2188T & 8 & 4.9 \\
\hline EYS & ex44 & c. $8868 \mathrm{C}>\mathrm{A}$ & p.Y2956 ${ }^{*}$ & 22 & 13.4 \\
\hline
\end{tabular}

${ }^{*}$ Truncating and nonsense variants.

(I)

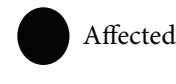

(II)

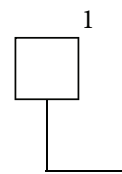

1

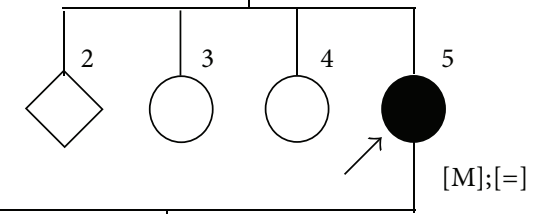

(III)
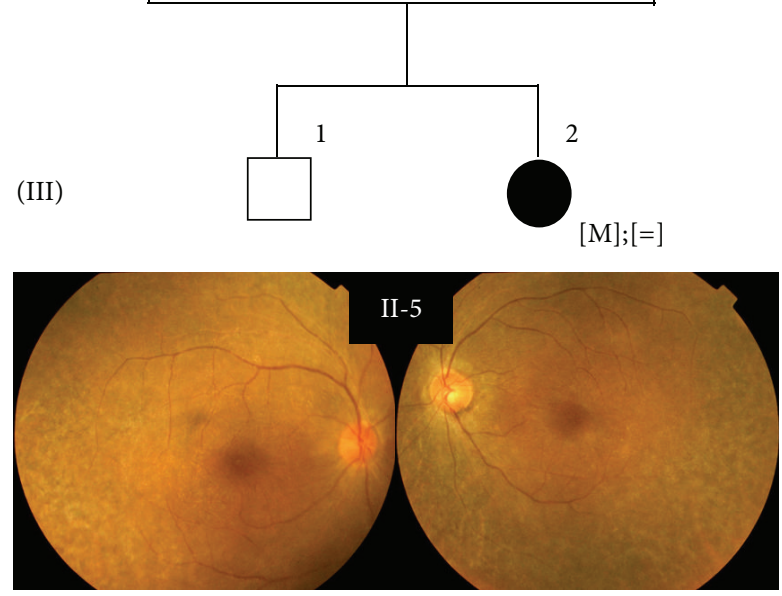

RHO ex1 c.36deLC hetero

FIGURE 4: A pedigree of probable adRP with a novel $R H O$ mutation. A family with a novel RHO mutation c.36delC is shown. II-5 in this family carried the heterozygote mutation, and her clinical phenotype was relatively mild with late onset at her age of 62. III2 showed only marginal clinical signs of RP when she underwent clinical evaluations at the age of 44 . A possible carrier of I-1 died before the age when the III-2 presented RP symptoms. Affected individuals are indicated as filled symbols, and an arrow indicates the proband in this family. Bottom images are fundus pictures of II5 .

found that mutations in EYS are the major cause of arRP in Spanish [21], Chinese and British [22], and Israeli and Palestinian populations [23]. In contrast, prevalence of EYS mutations was lower in other ethnic groups, and they account for approximately $5 \%$ of arRP patients of Western European ancestry [24]. These two major mutations, c.4957_4958insA and c.8868C $>$ A, have been only found in Japanese and Korean populations [11].

3.4.1. Association of EYS, CRB1, and PROM1 in Retinal Dystrophies. EYS was identified in Drosophila mutants with
(I)

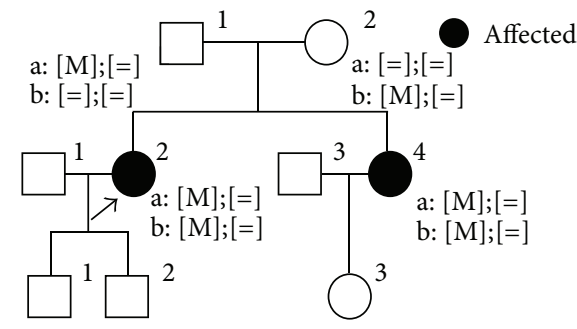

a: EYS c.8439_8442dupTGCA

b: EYS c.5202_5203delGT

FIGURE 5: A pedigree of arRP with novel EYS mutations. A family carrying two novel EYS mutations, c.8439_8442dupTGCA (a) and c.5202_5203delGT (b), is presented. Affected II-2 and II4 carried compound heterozygous mutations. I-1 carried a heterozygous c.8439_8442dupTGCA mutation and I-2 did another c.5202_5203delGT heterozygous mutation. Affected individuals are indicated as filled symbols, and an arrow indicates the proband in this family.

a compromised optomotor response [25] and characterized as a molecule which plays an important role for producing an interrhabdomeral space in the Drosophila retina [26]. Recent study with Drosophila models revealed that eys forms a genetic network with chaoptin, prominin, and crumbs for controlling the apical compartment of their photoreceptor cells [27]. Interestingly, the same study reported that deficiency of eys, prominin, or crumbs in flies displayed lightinduced photoreceptor degeneration. Furthermore, lightinduced photoreceptor death in these three mutants was rescued by culturing under vitamin A deficient conditions. Mutations of human homologs of these three genes, EYS [12], PROM1 [28], and CRB1 [29], can cause RP. All of these observations led us to speculate that RP caused by these genes could share disease pathogenesis. Simultaneously such observations also suggest possible digenic and polygenic diseases due to combination(s) of their impairments as observed in ROM1 and RDS/PRPH2 mutations [30].

Therefore, we examined the association of these three genes, EYS, PROM1, and CRB1, in $12 \mathrm{RP}$ patients with a heterozygous EYS mutation and two family members (Table 7). This additional screening found a novel heterozygous probable pathogenic PROM1 mutation c.1738A >C in two patients. Notably, PROM1 mutations have been reported to cause autosomal dominant cone dystrophy [31], and, indeed, the heterozygous mutation was found in our autosomal dominant cone dystrophy (Table 3). PROM1 mutations can cause 
TABle 7: Association of EYS, PROM1, and CRB1 in retinal dystrophies.

\begin{tabular}{lccc}
\hline EYS & PROM1 & CRB1 & Diagnosis \\
\hline c.768A $>$ G (p.I256M) & c.1738A $>$ C (p.N580H) & c.2306G $>$ A (p.R769H) & adRP \\
c.4957_4958insA & c.1738A $>$ C (p.N580H) & c.2306G $>$ A (p.R769H) & adRP \\
c.1450G $>$ A (p.G484R) & n.d. & c.2306G $>$ A (p.R769H) & adRP \\
c.8868C $>$ A (p.Y2956 $\left.{ }^{*}\right)$ & n.d. & c.2306G $>$ A (p.R769H) & adRP \\
c.4963T $>$ A (p.L1655M) & n.d. & c.2306G $>$ A (p.R769H) & arRP \\
\hline n.d. & n.d. & c.2306G $>$ A (p.R769H) & $($ Normal) \\
\hline
\end{tabular}

12 patients with RP were examined.

* Truncating and nonsense variants.

adRP indicates likely autosomal dominant retinitis pigmentosa.

n.d. indicates not detected.

arRP [32]. Furthermore, these two patients also carried a CRB1 sequence variant c.2306G $>A$ (rs62636287 in NCBI database) whereas 1,208 control subjects did not. c.2306G $>A$ was also found in a healthy family member without carrying a heterozygous EYS mutation. In the neighboring area of c. $2306 \mathrm{G}>\mathrm{A}$, c. $2302 \mathrm{C}>\mathrm{T}$ an unreported variant, c. $2303 \mathrm{G}>\mathrm{A}$, and c.2303C $>\mathrm{T}$ reported nonpathogenic variants were identified in 2, 6, and 3 control individuals, respectively. Moreover, 5 of 12 patients carried c. $2306 \mathrm{G}>\mathrm{A}$ in addition to a heterozygous EYS mutation. These results suggest the possibility of digenic and polygenic diseases due to combination(s) of EYS, PROM1, and CRB1 mutations. It is worth noting that copy number variants (CNVs) in EYS were reported in patients with IRD [33], and, therefore, molecular diagnosis of EYS should include analysis for CNVs. Our study observations and the relatively high prevalence of known recessive RP mutations in the general population [34] could produce additional complexity and difficulty in genetic counseling for arRP cases.

\section{Conclusion}

Molecular diagnosis of patients from different ethnic backgrounds greatly contributes to our understanding of the global spectrum of human disease-causing mutations and helps to develop therapies. Considering the possible number of therapeutic targets and relatively small number of patient populations with IRD, it is essential to define which genes can be the candidates for developing new therapies and which genes could more efficiently bring benefits to more patients worldwide. This study revealed different patterns of disease and genetic prevalence in Japanese populations as compared to European and North American populations. Most significantly Japanese populations have a higher prevalence of EYS mutations (23.5\%) and less rhodopsin mutations (2.0\%) compared to European and North American populations. In our Japanese cohort, we did not find the most common European origin $\mathrm{P} 23 \mathrm{H}$ of $\mathrm{RHO}$ mutation in adRP [35]. Additionally, we found a higher rate of RP and a lower prevalence of Stargardt disease in this Japanese cohort. Drawbacks of this study exist including patient referral bias and the heterogeneous disease presentation due to the wide range of clinical phenotypes seen in Stargardt disease $[36,37]$ and other IRD; nevertheless the data presented here demonstrates clear differences in causative genetic mutations between racial backgrounds.

In order to further increase the rate of identification of pathogenic mutations in IRD, other methods could be employed, such as entire exome sequencing using next generation sequencing (NGS), which utilizes a greater genetic panel for screening. Currently, both targeted and whole exome or genome analyses combined with NGS are becoming prevalent and several analyzers on the market reduce both time of analysis and cost of sequencing [38-40]. Because such NGS can also be easily shifted to and combined with RNA sequence analysis, understanding of pathogenesis due to specific mutations can be vastly improved. Furthermore, this study and other studies suggest that EYS screening for all $\mathrm{RP}, R P G R$, and RP2 for xlRP and male simplex RP [9] should be included as first tier assessment for Japanese patients with RP.

In summary, we performed the largest comprehensive mutational analysis on IRD in a Japanese population cohort. We identified 205 sequence variants in 349 probands and found a high prevalence of mutations in EYS. Furthermore, this is the first study examining a spectrum of IRD in Japanese cases and it provided additional evidence of differential disease prevalence among races.

\section{Abbreviations}

ABCA4: ATP-binging cassette transporter, family 4

CHM: Choroideremia gene

CYP4V2: Cytochrome P450, family 4, subfamily V, polypeptide 2

EYS: $\quad$ Eyes shut homolog

IRD: Inherited retinal dystrophy

PRPH2: Peripherin 2

RDH5: Retinol dehydrogenase 5

RDS: Retinal degeneration slow

RHO: Rhodopsin

RLBP1: Retinol binding protein 1

RP: $\quad$ Retinitis pigmentosa

RS1: Retinoschisis 1

SAG: S-antigen or arrestin

USH2A: Usher syndrome 2A

VMD2: Vitelliform macular dystrophy 2. 


\section{Conflict of Interests}

The authors declare that there is no conflict of interests regarding the publication of this paper.

\section{Acknowledgments}

The authors thank all individuals who participated in this study. This work was supported by funding from the Research Center Network for Realization of Regenerative Medicine (MEXT for MT) and the NIH (EY022658 for AM). They are grateful for Dr. Elias I. Traboulsi (Cleveland Clinic, Cleveland, OH), Dr. Norimoto Gotoh (Kyoto University, Kyoto, Japan), Drs. Tanu Parmar and Lindsay Perusek (Case Western Reserve University), and Kanako Kawai and Tomoko Yokota (RIKEN) for their comments and technical support.

\section{References}

[1] S. P. Daiger, L. S. Sullivan, and S. J. Bowne, "Genes and mutations causing retinitis pigmentosa," Clinical Genetics, vol. 84, no. 2, pp. 132-141, 2013.

[2] A. I. den Hollander, A. Black, J. Bennett, and F. P. M. Cremers, "Lighting a candle in the dark: advances in genetics and gene therapy of recessive retinal dystrophies," Journal of Clinical Investigation, vol. 120, no. 9, pp. 3042-3053, 2010.

[3] S. Roosing, A. A. Thiadens, C. B. Hoyng, C. C. Klaver, A. I. den Hollander, and F. P. Cremers, "Causes and consequences of inherited cone disorders," Progress in Retinal and Eye Research, vol. 42, no. 9, pp. 1-26, 2014.

[4] Z.-B. Jin, M. Mandai, T. Yokota et al., "Identifying pathogenic genetic background of simplex or multiplex retinitis pigmentosa patients: a large scale mutation screening study," Journal of Medical Genetics, vol. 45, no. 7, pp. 465-472, 2008.

[5] M. Narahara, K. Higasa, S. Nakamura et al., "Large-scale EastAsian eQTL mapping reveals novel candidate genes for LD mapping and the genomic landscape of transcriptional effects of sequence variants," PLoS ONE, vol. 9, no. 6, Article ID e100924, 2014.

[6] M. A. Genead, G. A. Fishman, E. M. Stone, and R. Allikmets, "The natural history of stargardt disease with specific sequence mutation in the ABCA4 gene," Investigative Ophthalmology \& Visual Science, vol. 50, no. 12, pp. 5867-5871, 2009.

[7] R. B. Welch, "Bietti's tapetoretinal degeneration with marginal corneal dystrophy crystalline retinopathy," Transactions of the American Ophthalmological Society, vol. 75, pp. 164-179, 1977.

[8] E. M. Stone, A. J. Aldave, A. V. Drack et al., "Recommendations for genetic testing of inherited eye diseases: report of the American academy of ophthalmology task force on genetic testing," Ophthalmology, vol. 119, no. 11, pp. 2408-2410, 2012.

[9] K. Branham, M. Othman, M. Brumm et al., "Mutations in RPGR and RP2 account for $15 \%$ of males with simplex retinal degenerative disease," Investigative Ophthalmology and Visual Science, vol. 53, no. 13, pp. 8232-8237, 2012.

[10] M. Iwanami, M. Oshikawa, T. Nishida, S. Nakadomari, and S. Kato, "High prevalence of mutations in the EYS gene in japanese patients with autosomal recessive retinitis pigmentosa," Investigative Ophthalmology \& Visual Science, vol. 53, no. 2, pp. 10331040, 2012.

[11] K. Hosono, C. Ishigami, M. Takahashi et al., "Two novel mutations in the EYS gene are possible major causes of autosomal recessive retinitis pigmentosa in the japanese population," $P L o S$ ONE, vol. 7, no. 2, Article ID e31036, 2012.

[12] M. M. Abd El-Aziz, I. Barragan, C. A. O’Driscoll et al., "EYS, encoding an ortholog of Drosophila spacemaker, is mutated in autosomal recessive retinitis pigmentosa," Nature Genetics, vol. 40, no. 11, pp. 1285-1287, 2008.

[13] Y. Zhao, K. Hosono, K. Suto et al., "The first USH2A mutation analysis of Japanese autosomal recessive retinitis pigmentosa patients: a totally different mutation profile with the lack of frequent mutations found in Caucasian patients," Journal of Human Genetics, vol. 59, no. 9, pp. 521-528, 2014.

[14] M. Oishi, A. Oishi, N. Gotoh et al., "Comprehensive molecular diagnosis of a large cohort of Japanese retinitis pigmentosa and usher syndrome patients by next-generation sequencing," Investigative Ophthalmology \& Visual Science, vol. 55, no. 11, pp. 7369-7375, 2014.

[15] M. Sato, M. Nakazawa, T. Usui, N. Tanimoto, H. Abe, and H. Ohguro, "Mutations in the gene coding for guanylate cyclaseactivating protein 2 (GUCA1B gene) in patients with autosomal dominant retinal dystrophies," Graefe's Archive for Clinical and Experimental Ophthalmology, vol. 243, no. 3, pp. 235-242, 2005.

[16] A. M. Payne, S. M. Downes, D. A. R. Bessant et al., "Genetic analysis of the guanylate cyclase activator $1 B$ (GUCA1B) gene in patients with autosomal dominant retinal dystrophies," Journal of Medical Genetics, vol. 36, no. 9, pp. 691-693, 1999.

[17] V. B. D. Kitiratschky, C. J. Glöckner, and S. Kohl, "Mutation screening of the GUCA1B gene in patients with autosomal dominant cone and cone rod dystrophy," Ophthalmic Genetics, vol. 32, no. 3, pp. 151-155, 2011.

[18] A. Ruiz, S. Borrego, I. Marcos, and G. Antiñolo, "A major locus for autosomal recessive retinitis pigmentosa on 6q, determined by homozygosity mapping of chromosomal regions that contain gamma- aminobutyric acid-receptor clusters," American Journal of Human Genetics, vol. 62, no. 6, pp. 1452-1459, 1998.

[19] S. Khaliq, A. Hameed, M. Ismail et al., "Refinement of the locus for autosomal recessive retinitis pigmentosa (RP25) kinked to chromosome 6q in a family of pakistani origin," The American Journal of Human Genetics, vol. 65, no. 2, pp. 571-574, 1999.

[20] M. M. Abd El-aziz, M. F. El-ashry, W. M. Chan et al., "A novel genetic study of chinese families with autosomal recessive retinitis pigmentosa," Annals of Human Genetics, vol. 71, no. 3, pp. 281-294, 2007.

[21] I. Barragán, S. Borrego, J. I. Pieras et al., "Mutation spectrum of EYS in Spanish patients with autosomal recessive retinitis pigmentosa," Human Mutation, vol. 31, no. 11, pp. E1772-E1800, 2010.

[22] M. M. A. El-Aziz, C. A. O’Driscoll, R. S. Kaye et al., "Identification of novel mutations in the ortholog of Drosophila eyes shut gene (EYS) causing autosomal recessive retinitis pigmentosa," Investigative Ophthalmology \& Visual Science, vol. 51, no. 8, pp. 4266-4272, 2010.

[23] D. Bandah-Rozenfeld, K. W. Littink, T. Ben-Yosef et al., "Novel null mutations in the EYS gene are a frequent cause of autosomal recessive retinitis pigmentosa in the Israeli population," Investigative Ophthalmology \& Visual Science, vol. 51, no. 9, pp. 4387-4394, 2010.

[24] K. W. Littink, L. I. van den Born, R. K. Koenekoop et al., "Mutations in the EYS gene account for approximately 5\% of autosomal recessive retinitis pigmentosa and cause a fairly homogeneous phenotype," Ophthalmology, vol. 117, no. 10, pp. 2026.e7-2033.e7, 2010. 
[25] T. R. Clandinin, C.-H. Lee, T. Herman et al., "Drosophila LAR regulates R1-R6 and R7 target specificity in the visual system," Neuron, vol. 32, no. 2, pp. 237-248, 2001.

[26] N. Husain, M. Pellikka, H. Hong et al., "The agrin/perlecanrelated protein eyes shut is essential for epithelial lumen formation in the Drosophila retina," Developmental Cell, vol. 11, no. 4, pp. 483-493, 2006.

[27] N. Gurudev, M. Yuan, and E. Knust, "Chaoptin, prominin, eyes shut and crumbs form a genetic network controlling the apical compartment of Drosophila photoreceptor cells," Biology Open, vol. 3, no. 5, pp. 332-341, 2014.

[28] M. A. Maw, D. Corbeil, J. Koch et al., "A frameshift mutation in prominin (mouse)-like 1 causes human retinal degeneration," Human Molecular Genetics, vol. 9, no. 1, pp. 27-34, 2000.

[29] A. I. den Hollander, J. B. ten Brink, Y. J. M. de Kok et al., "Mutations in a human homologue of Drosophila crumbs cause retinitis pigmentosa (RP12)," Nature Genetics, vol. 23, no. 2, pp. 217-221, 1999.

[30] K. Kajiwara, E. L. Berson, and T. P. Dryja, "Digenic retinitis pigmentosa due to mutations at the unlinked peripherin/RDS and ROM1 loci," Science, vol. 264, no. 5165, pp. 1604-1608, 1994.

[31] M. Kniazeva, M. F. Chiang, B. Morgan et al., "A new locus for autosomal dominant Stargardt-like disease maps to chromosome 4," The American Journal of Human Genetics, vol. 64, no. 5, pp. 1394-1399, 1999.

[32] Q. Zhang, F. Zulfiqar, X. Xiao et al., "Severe retinitis pigmentosa mapped to 4 p15 and associated with a novel mutation in the PROM1 gene," Human Genetics, vol. 122, no. 3-4, pp. 293-299, 2007.

[33] T. Eisenberger, C. Neuhaus, A. O. Khan et al., "Increasing the yield in targeted next-generation sequencing by implicating $\mathrm{CNV}$ analysis, non-coding exons and the overall variant load: the example of retinal dystrophies," PLoS ONE, vol. 8, no. 11, Article ID e78496, 2013.

[34] K. M. Nishiguchi and C. Rivolta, "Genes associated with retinitis pigmentosa and allied diseases are frequently mutated in the general population," PLoS ONE, vol. 7, no. 7, Article ID e41902, 2012.

[35] T. P. Dryja, T. L. McGee, L. B. Hahn et al., "Mutations within the rhodopsin gene in patients with autosomal dominant retinitis pigmentosa," The New England Journal of Medicine, vol. 323, no. 19, pp. 1302-1307, 1990.

[36] T. Fukui, S. Yamamoto, K. Nakano et al., "ABCA4 gene mutations in Japanese patients with Stargardt disease and retinitis pigmentosa," Investigative Ophthalmology \& Visual Science, vol. 43, no. 9, pp. 2819-2824, 2002.

[37] S. Walia and G. A. Fishman, "Natural history of phenotypic changes in Stargardt macular dystrophy," Ophthalmic Genetics, vol. 30, no. 2, pp. 63-68, 2009.

[38] K. Neveling, R. W. J. Collin, C. Gilissen et al., "Next-generation genetic testing for retinitis pigmentosa," Human Mutation, vol. 33, no. 6, pp. 963-972, 2012.

[39] J. O’Sullivan, B. G. Mullaney, S. S. Bhaskar et al., "A paradigm shift in the delivery of services for diagnosis of inherited retinal disease," Journal of Medical Genetics, vol. 49, no. 5, pp. 322-326, 2012.

[40] M. E. Shanks, S. M. Downes, R. R. Copley et al., "Nextgeneration sequencing (NGS) as a diagnostic tool for retinal degeneration reveals a much higher detection rate in early-onset disease," European Journal of Human Genetics, vol. 21, no. 3, pp. 274-280, 2013. 


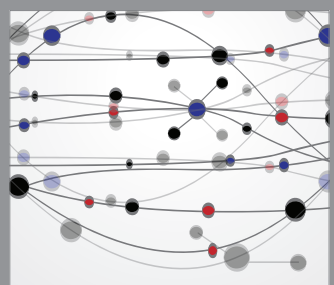

The Scientific World Journal
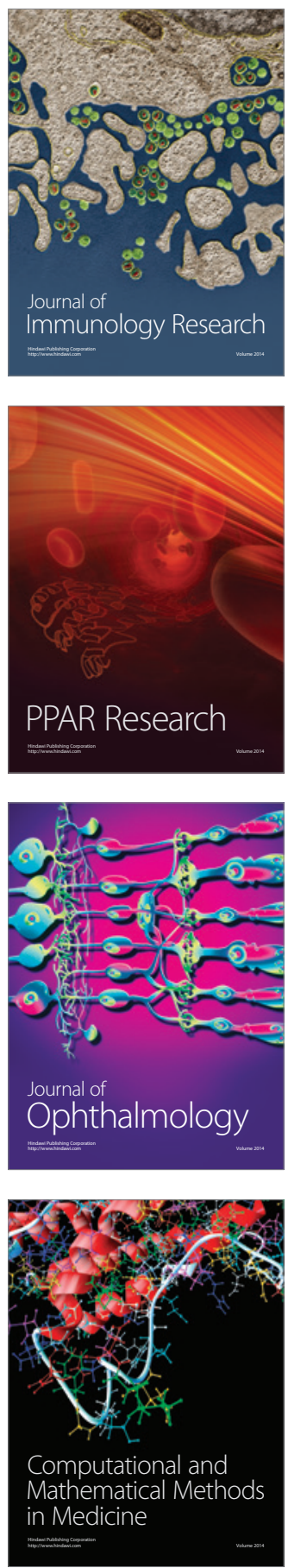

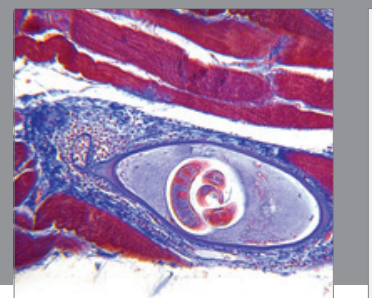

Gastroenterology

Research and Practice
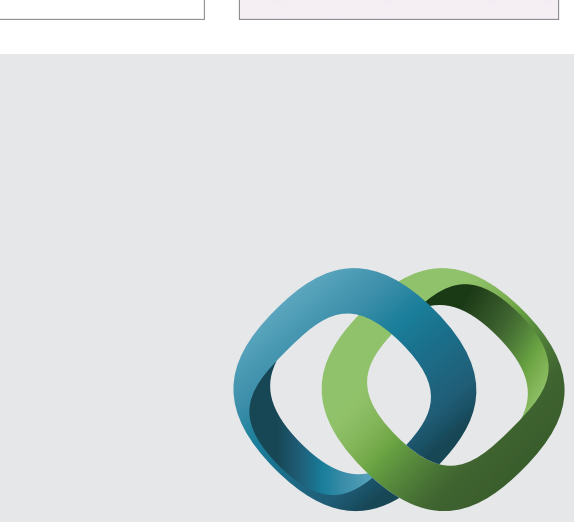

\section{Hindawi}

Submit your manuscripts at

http://www.hindawi.com
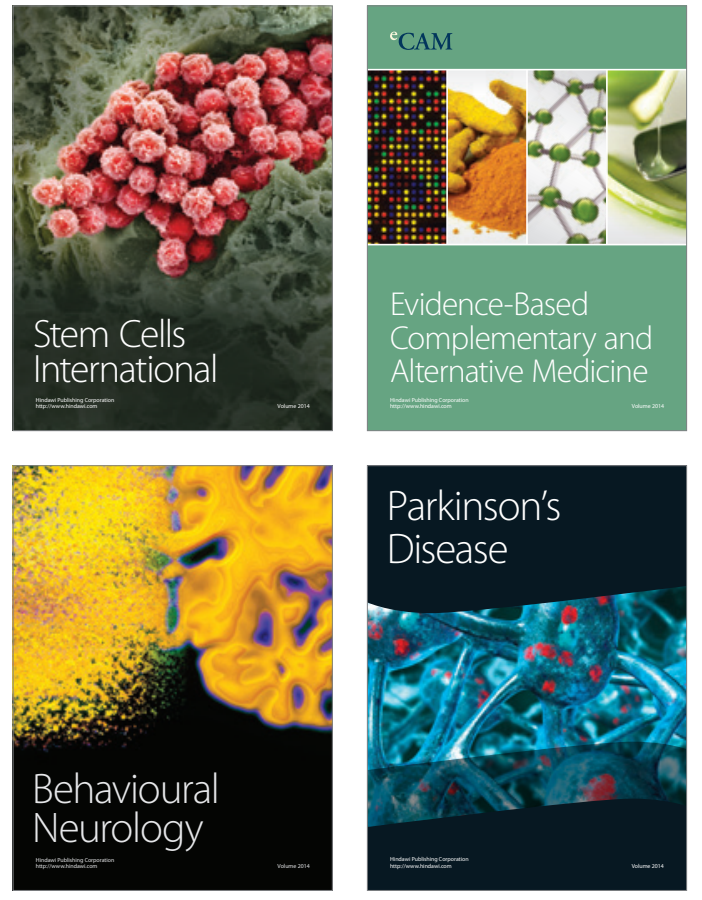
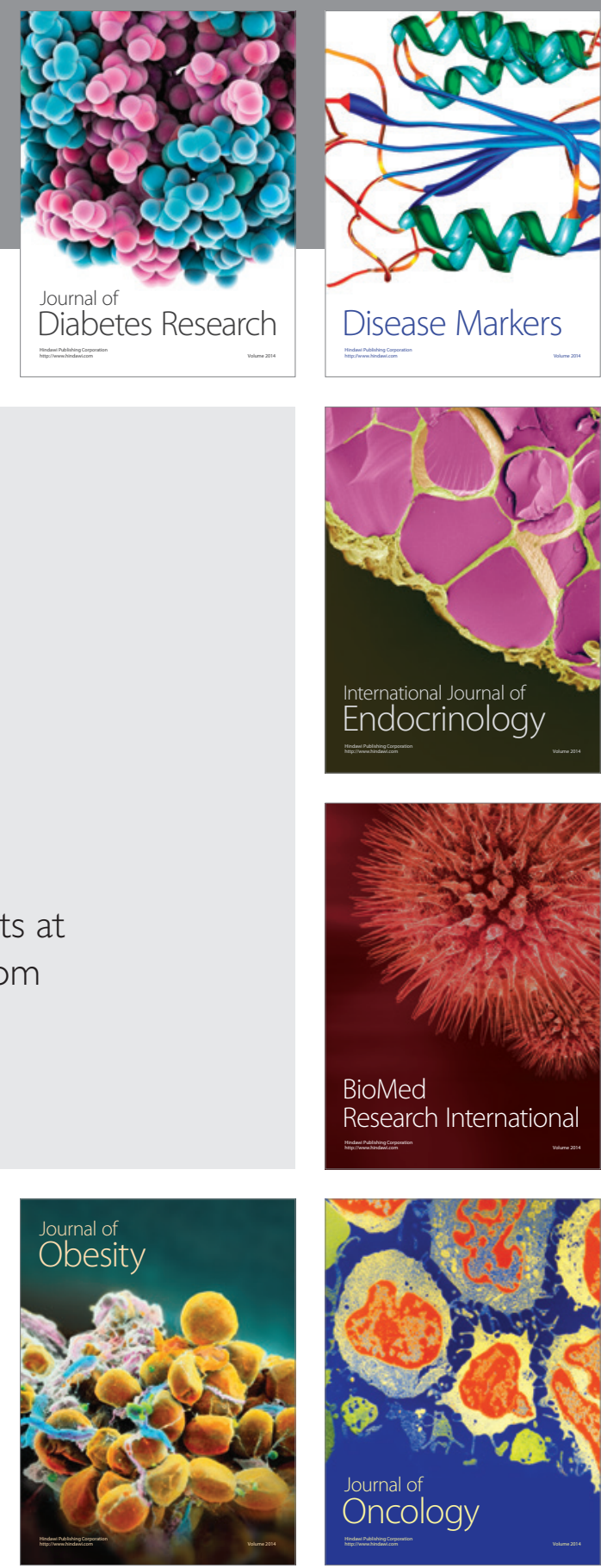

Disease Markers
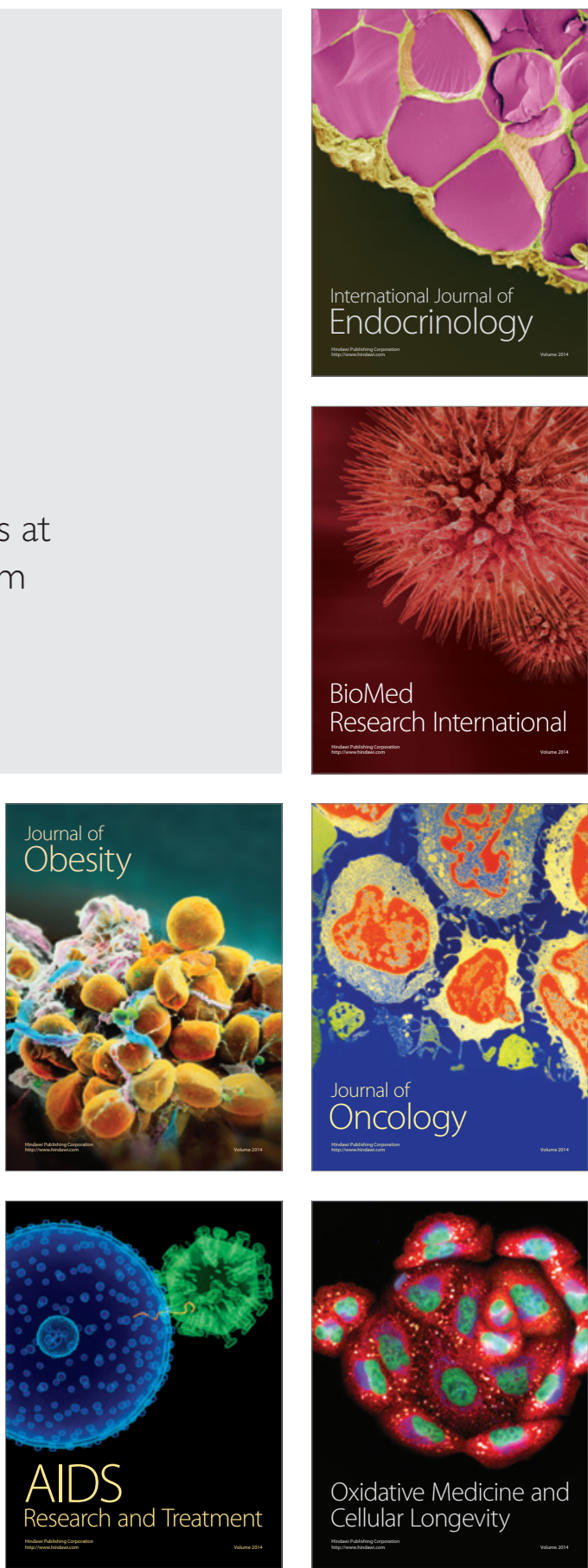